\title{
Social chromosome variants differentially affect queen determination and the survival of workers in the fire ant Solenopsis invicta
}

\author{
SÉVERINE D. BUECHEL ${ }^{*} \dagger^{1}$ YANNICK WURM* ${ }^{*}$ and LAURENT KELLER* \\ *Department of Ecology and Evolution, University of Lausanne, Sorge, Le Biophore, CH-1015 Lausanne, Switzerland, $\dagger$ Institute \\ of Integrative Biology (IBZ), ETH Zürich, Universitätsstrasse 16, CH-8092 Zürich, Switzerland, $¥$ School of Biological and \\ Chemical Sciences, Queen Mary, University of London, London E1 4NS, UK
}

\begin{abstract}
Intraspecific variation in social organization is common, yet the underlying causes are rarely known. An exception is the fire ant Solenopsis invicta in which the existence of two distinct forms of social colony organization is under the control of the two variants of a pair of social chromosomes, $\mathrm{SB}$ and $\mathrm{Sb}$. Colonies containing exclusively SB/SB workers accept only one single queen and she must be SB/SB. By contrast, when colonies contain more than $10 \%$ of $\mathrm{SB} / \mathrm{Sb}$ workers, they accept several queens but only $\mathrm{SB} / \mathrm{Sb}$ queens. The variants of the social chromosome are associated with several additional important phenotypic differences, including the size, fecundity and dispersal strategies of queens, aggressiveness of workers, and sperm count in males. However, little is known about whether social chromosome variants affect fitness in other life stages. Here, we perform experiments to determine whether differential selection occurs during development and in adult workers. We find evidence that the $\mathrm{Sb}$ variant of the social chromosome increases the likelihood of female brood to develop into queens and that adult $\mathrm{SB} / \mathrm{Sb}$ workers, the workers that cull $\mathrm{SB} / \mathrm{SB}$ queens, are overrepresented in comparison to SB/SB workers. This demonstrates that supergenes such as the social chromosome can have complex effects on phenotypes at various stages of development.
\end{abstract}

Keywords: fire ants, Gp-9, ontogenetic selection, social chromosome, social evolution, Solenopsis invicta

Received 27 July 2014; revision received 29 August 2014; accepted 29 August 2014

\section{Introduction}

Intraspecific variation in social organization is common, yet the underlying causes are rarely known. An exception is the fire ant Solenopsis invicta in which the existence of two distinct forms of social colony organization is under the control of a single Mendelian genomic element (Keller \& Ross 1998; Ross \& Keller 1998). Colonies of the monogyne form are headed by a single reproductive queen, whereas colonies of the polygyne

Correspondence: Séverine D. Buechel, Department of Zoology/ Ethology, Stockholm University, Svante Arrhenius väg 18B, SE10691 Stockholm, Sweden, E-mail: severine.buechel@zoologi.su.se ${ }^{1}$ These authors contributed equally to this work. form contain multiple such queens (Ross \& Keller 1995). Population genetic studies revealed a perfect association between social organization and the genotypic composition of colony members at the proteincoding gene $\mathrm{Gp}$-9. In monogyne colonies, the queen and all workers are invariably homozygous for the $G p-9^{B}$ allele (Ross 1997). By contrast, polygyne colonies contain only queens with a $G p-9^{B b}$ genotype and a mixture of $G p-9^{B B}$ and $G p-9^{B b}$ workers (Ross 1997). The virtual absence of $G p-9^{b b}$ queens and workers in polygyne colonies is due to $G p-9^{b}$ behaving as a lethal recessive allele in females, inducing their death soon after they eclose from the pupae (Ross 1997; Keller \& Ross 1999; Hallar et al. 2007). Experimental studies also revealed that the complete absence of $G p-9^{B B}$ queens in polygyne colonies 
is due to the $G p-9^{b}$ allele being linked to a selfish genetic element inducing $G p-9^{B b}$ workers to selectively eliminate $G p-9^{B B}$ queens when they initiate reproduction (Keller \& Ross 1998). Thus, when colonies contain more than approximately $10 \% G p-9^{B b}$ workers, they accept several queens, but only $G p-9^{B b}$ queens (Ross \& Keller 2002; Gotzek \& Ross 2008). By contrast, when colonies contain only $G p-9^{B B}$ workers, they only accept a single $G p-9^{B B}$ queen (Ross \& Keller 1998).

In addition to social organization, the Gp-9 genotype is also associated with several important phenotypic differences. For instance, young (prereproductive) $G p-9^{B B}$ queens (from both monogyne and polygyne colonies) store large fat reserves after eclosion from the pupae and undertake long-distance mating flights prior to founding colonies independently, whereas $G p-9^{B b}$ queens typically store few reserves and undertake only localized mating flights before attempting to infiltrate existing colonies to begin reproducing (Keller \& Ross 1993a,b, 1999; DeHeer et al. 1999; Goodisman et al. 2000a; DeHeer 2002). Haploid males of alternate Gp-9 genotype also exhibit phenotypic differences with $G p-9^{b}$ males having significantly less sperm than $G p-9^{B}$ males (Lawson et al. 2012).

While there is considerable information on the different processes of selection acting on adult queens and workers with alternate Gp-9 genotypes (Keller \& Ross 1993b, 1998; DeHeer et al. 1999; Goodisman et al. 1999, 2007; DeHeer 2002; Krieger 2005), little is known about possible differences in fitness in other life stages. Field surveys of workers and brood from polygyne colonies suggest that the relative frequencies of the $G p-9^{B}$ and $G p-9^{b}$ alleles differ from those expected under Mendelian, [Table S1 (Supporting information) summarizes results from Ross (1997); Goodisman et al. (2000b) and Fritz (2006)], but field surveys may include sources of bias that are difficult to account for.

Given the numerous effects associated with Gp-9 genotype in adults, it is conceivable that there is differential selection on immature individuals with alternate $G p-9$ genotypes. This is particularly true because Gp-9 was recently found to be in linkage disequilibrium with more than 600 other genes. Indeed, the two variants of the social chromosome (i.e. the social b chromosome which contains the $G p-9^{b}$ allele and the social B chromosome which contains the $G p-9^{B}$ allele) do not recombine with one another over a large genomic region $[13.8 \mathrm{Mb}$, $55 \%$ of the chromosome; (Wang et al. 2013)]. Furthermore, numerous SNPs are fixed between the two social chromosome variants (Wang et al. 2013), and there is an overrepresentation of genes differently expressed between individuals of alternative genotype that reside in the nonrecombining region (Wang et al. 2008; Nipitwattanaphon et al. 2013; Wang et al. 2013). It is therefore likely that individuals with alternate Gp-9 genotypes have multiple phenotypic differences that may affect the process of development.

Detailed studies of selection acting on fire ants with alternate $G p-9$ genotypes is of special interest given the increasing evidence that supergenes (i.e. tight clusters of two or more loci each affecting a different developmental or behavioural characteristic) play an important role in maintaining the cosegregation of adaptive variation within species (reviewed in Schwander et al. 2014). Recent studies have led to the discovery of a large panoply of phenotypes being regulated by supergenes in plants, insects, birds, fishes and mammals (reviewed in Schwander et al. 2014). In some of the best studied cases, such as in the white-throated sparrow, the supergene is known to affect phenotypic traits as diverse as plumage colour, song and courtship behaviour (Thomas et al. 2008). However, in the case of the white-throated sparrow, as well as the other cases of supergenes, there is generally very little data on how variants of the supergenes affect fitness and almost no information on how patterns of selection vary throughout development.

To test whether differential selection on $G p-9^{B B}$ and $G p-9^{B b}$ S. invicta females occurs during development, we isolated $G p-9^{B b}$ queens mated with a $G p-9^{B}$ male and transferred their eggs into queenless colonies containing either monogyne or polygyne workers. After several weeks of development, we collected queen and worker pupae to test for deviation from the expected value of $50 \% \quad G p-9^{B B}$ and $50 \% \quad G p-9^{B b}$ individuals. Because many aspects of social life are affected by the presence or absence of a queen in fire ants and other ants (e.g. Keller 1995; Ross \& Keller 1995), we also followed the development of brood in colonies containing a queen. Finally, to test whether potential effects on development affected $\mathrm{G} p-9$ genotype distribution among adult workers, we collected and measured the size of ants inside and outside the nest, and genotyped them in proportion to their size and occurrence in each location.

\section{Materials and methods}

\section{Collection and rearing of study colonies}

Queenright polygyne and monogyne colonies of S. invicta were collected near Athens, Georgia (USA), in March 2008. Colonies were transferred to the laboratory and maintained under standard conditions (Jouvenaz et al. 1977). All colonies were fed on a daily basis with a high-protein diet (tuna/vegetable/peanut butter mix) supplemented by crickets, mealworms or cockroaches. The monogyne status of colonies was inferred upon 
collection by the presence of a single highly physogastric, wingless queen per colony. The polygyne status of the colony was inferred from the presence of multiple wingless queens. Social form of all used colonies was always later confirmed by $G p-9$ genotyping at least 50 young winged queens per colony. All polygyne colonies were found to include young $G p-9^{B b}$ queens, while no such queens were found in any monogyne colony (Nipitwattanaphon et al. 2013).

\section{Gp-9 specific rearing of female brood}

To determine whether Gp-9 genotypes were skewed among female pupae, we first selected $G p-9^{B b}$ queens [the type of queen most common in polygyne colonies, (Ross 1997)] mated with a $G p-9^{B}$ male. For this, we collected five queens from each of 10 polygyne donor colonies and placed them with workers in small units for 80 days (mean; range of 42-100 days). To ensure that only experimental queens sired pupae, an isolation period of 35 days is essential because development from egg to adult takes approximately 35 days in standard $28{ }^{\circ} \mathrm{C}$ conditions (Porter 1988). We then collected 10 worker pupae from each unit, genotyped them at Gp-9 and selected the queens that exclusively produced $B B$ and $B b$ brood (i.e. $G p-9^{B b}$ queens mated to $G p-9^{B}$ males).

We first transferred eggs from 10 such queens (one per donor colony) into 10 monogyne and 10 polygyne recipient queenless colonies each containing approximately 5000 workers originating from either a monogyne colony or a polygyne colony. All recipient colonies originated from laboratory colonies that were not used in other experiments, and each of them was held queenless for 42 days prior to the experiments to ensure that they contained only adult workers. To obtain eggs, each of the donor queens was isolated twice for $48 \mathrm{~h}$ with 5-10 workers from her colony. One batch of eggs was introduced into a recipient monogyne colony and the other batch of eggs into a recipient polygyne colony. This egg introduction was balanced with regards to the sequence of egg introduction into recipient colonies of the two alternate social forms. Twenty-eight days after egg introduction, all worker and queen pupae were collected from the recipient colonies for genotyping at $G p-9$.

To study whether the presence/absence of queens affects the differential survival of $G p-9^{B B}$ and $G p-9^{B b}$ brood, we then selected two $G p-9^{B b}$ queens each mated with one $G p-9^{B}$ male from each of 10 polygyne colonies and introduced one of them into a recipient monogyne colony kept queenless for 40 days and the other into a recipient polygyne colony kept queenless for 40 days (the queens introduced were a subset of those used to produce the eggs in the previous experiment; queens were introduced with some brood from their colony to increase the probability of acceptance by the workers). All recipient colonies were created from source colonies that were not otherwise used for this study. Queen introductions followed the protocol described in Ross \& Keller (2002) with minor modifications. In brief, recipient workers were chilled at $4{ }^{\circ} \mathrm{C}$ before introducing the new queen and her brood. Human breath was blown onto the queen and nearby workers, and water mist was sprayed onto the workers when they showed aggression towards the queen. All worker pupae were sampled 42 days after queen introduction. Because only a few queen pupae were present in the colonies at this time, we continued to sample queen pupae over a period of three weeks to obtain sufficient numbers of individuals for the analyses.

Queen pupae were reared in three of the 10 queenless polygyne recipient colonies, three of the 10 queenless monogyne recipient colonies, five of the 10 queenright polygyne recipient colonies and none of the 10 queenright monogyne recipient colonies. The high proportion of colonies that produced no queens probably reflects the fact that there is strong sex ratio specialization in fire ants, with many colonies producing no sexuals or only male or only female sexuals (Aron et al. 1995; Vargo 1996). This strong variation among colonies apparently stems from differences in the primary sex ratio laid by queens and colony variation in the likelihood of the workers to raise new queens and males (Aron et al. 1995; Passera et al. 2001; Tschinkel 2006). However, the proportion of colonies that raised new queens was not significantly different among the four types of colonies (monogyne queenless, monogyne queenright, polygyne queenless and polygyne queenright; Fisher's exact test: $n=40, P=0.08$ ), suggesting that the complete absence of queen pupae in queenright monogyne colonies may just be a sampling effect. We sampled all 336 queen pupae that were reared from both queenless and queenright recipient colonies and determined their $G p-9$ genotype. Among all the worker pupae that were reared, we sampled and genotyped a subsample of 561 pupae that were collected on a single day. This represents only a small fraction of all the pupae that were produced in the colonies. These worker pupae were collected from all 10 polygyne and all 10 monogyne queenless recipient colonies, but for time constraints, we only sampled and genotyped worker pupae in four of the 10 polygyne and four of the 10 monogyne queenright recipient colonies.

\section{Gp-9 genotype frequencies and association with worker size and location}

To determine whether the Gp-9 genotypes among adults deviated from the expected $50 \%$ and to account for a potential sampling bias due to an effect of $G p-9$ on 
worker location and/or worker size, we created six colonies each headed by a single $G p-9^{B b}$ queen mated with a $G p-9^{B}$ male (each queen originating from a different donor colony). Six months later [expected lifespan is approximately 51 days for minor workers and 121 days for major workers of S. invicta (Tschinkel 2006)], we genotyped 16 adult workers and eight larvae per colony at six microsatellite loci (see Microsatellite genotyping) to confirm that all workers were the progeny of the queen and that all queens were singly mated.

We distinguished between workers from inside the nest (i.e. with the queen and brood inside two $10 \mathrm{~cm}$ diameter plaster-bottom petri dishes) and workers from outside the nest (i.e. anywhere else in the $25 \mathrm{~cm}$ wide $\times 40 \mathrm{~cm}$ long rearing box) by removing the nest and placing it into an empty rearing box prior to collection. This procedure was used because small disturbances (e.g. researchers attempting to collect some ants) rapidly trigger excitement and an alarm-like status inducing workers to change location (Bradshaw \& Howse 1984). Ants from inside and from outside the nest were killed by exposure to ethyl acetate to facilitate the subsequent sieving of workers into three size classes using geological sieves (Rentsch, Haan, Germany): small ants $(<1 \mathrm{~mm}$ grid diameter), medium ants $(<2 \mathrm{~mm}$ grid diameter) and large ants ( $>2 \mathrm{~mm}$ grid diameter). We tested different sieves to split ants into three relatively homogenous intraclass body sizes before selecting the 1 and $2 \mathrm{~mm}$ grid sizes. For all six colonies, we weighed all ants from each of the six groups of workers (small, medium and large workers from inside the nest and small, medium large workers from outside the nest) to the nearest $\mathrm{mg}$, counted and weighed in triplicate approximately 100 ants per group (mean: 105, range: 50-165) and used the mean of the three ratios and the weight of the whole group to estimate the total number of individuals in each group. Subsequently, we sampled and determined the Gp-9 genotype of a total of 1152 workers (192 workers from each colony). Of those individuals, we only obtained a reliable genotype for 744 individuals because the exposure to euthanization with ethyl acetate led to extremely low DNA extraction yields (Dillon et al. 1996).

\section{Genotyping at Gp-9}

Genomic DNA was extracted from whole pupae using the BioSprint 96 workstation and the BioSprint 96 DNA Blood Kit (Qiagen, Hilden, Germany). To genotype individual pupae at the Gp-9 locus, we used a nested polymerase chain reaction (PCR) using GP9CLF2 (TCTCGA TTGGTGAAGTATCAAGT) and GP9CLR2 (CATGTCA ATACAAAAGAAAGCTG) as external primers and GP9CLF1 (GATACCGAACTACACAAAAATGGTTGC) and GP9CLR1 (TTAGAATCGGCGAGCACAGCTT) as internal primers, and subsequently performed restriction fragment length analysis with the enzymes BSM1 and BVE1 (Fermentas GmbH, Germany; C. Lucas \& M. Nicolas, unpublished method). Using this method, we had difficulties obtaining genotypes for adult workers because euthanization with ethyl acetate led to extremely low DNA extraction yields (Dillon et al. 1996). For adult workers, we therefore used more sensitive protocols for both DNA extraction and genotyping at Gp-9. To extract DNA from whole ant bodies, each ant was washed in distilled water, snap frozen and homogenized using a micropestle in a $1.5 \mathrm{~mL}$ microtube. We then added $50 \mu \mathrm{L}$ Tris ( $10 \mathrm{~mm}, \mathrm{pH}$ 8.6) and centrifuged each homogenate at $12054 \mathrm{~g}$ for $150 \mathrm{~s}$. Finally, we added $2.5 \mu \mathrm{L}$ dNTPs ( $25 \mathrm{~mm}$ ) to the supernatant and the samples were incubated for $15 \mathrm{~min}$ at $95^{\circ} \mathrm{C}$. Adding dNTPs may protect the DNA from degradation as the latter bind to phenolic components such as DNAse and RNAse. We genotyped adults based on alternative hybridization of $G p-9^{B}$ and $G p-9^{b}$-specific fluorescent probes (Shoemaker \& Ascunce 2010) in an Applied Biosystems 7900HT Fast Real-Time PCR System. Specifically, we used the Gp-9_139 probeset which is diagnostic for Gp-9 alleles in North America (Shoemaker \& Ascunce 2010). Fluorescence intensities plotted in an allelic discrimination plot using Applied Biosystems SDS version 2.4 allowed rapid reliable determination of genotypes, including from samples with low quantities of DNA that had yielded no result using the previous method based on enzymatic-digestion.

\section{Microsatellite genotyping}

To study Gp-9 genotype distribution among adult workers, we used six colonies each including only a single queen. We used six microsatellite markers to confirm that the workers in the colony were full sisters. We extracted genomic DNA from worker larvae and adults using the BioSprint 96 DNA Blood kit (Qiagen) and subsequently determined genotypes at six polymorphic microsatellite loci. For this, we used the following primer sets: Sol-42; Sol-49; Sol-55; Sol-11; Sol-20 (Krieger \& Keller 1997); and Sdag C536 (Ascunce et al. 2009). Each forward primer was labelled with a fluorescent marker (HEX, FAM or ATTO-550), and the six loci were amplified in two separate multiplex polymerase chain reactions (PCR) generally following the PCR preparation protocol of Shoemaker et al. (2006) but replacing Sol-6 and Sol-18 with Sdag C536 and using the cycling profile of Krieger \& Keller (1997). PCR products were sequenced on an Applied Biosystems ABI PRISM 3100 Genetic Analyser and analysed with the GENEMAPPER software. 


\section{Statistical analysis}

The distributions of $G p-9$ genotypes of worker pupae were analysed using a generalized linear mixed model (GLMMs) with a binomial error structure (logit link function). The full model used social form of recipient colonies (monogyne vs. polygyne) and queen presence (queenright vs. queenless) as fixed effects and source colony (colony of the queen that served as egg donor) as a random effect. Nonsignificant interaction terms are not shown because model selection was performed by backwards elimination of nonsignificant terms (Crawley 1993). Significant terms were analysed post hoc using Fisher's LSD procedure for pairwise comparisons. To analyse the distribution of $G p-9$ genotypes among adult workers, we ran a second binomial model, which used worker size (small, medium and large) and sampling location (inside vs. outside nest) as fixed effects and colony as a random effect. In a third binomial model, we examined the distribution of workers between size classes (small, medium and large) and sampling location (inside vs. outside the nest), in an analysis where colony was included as a random effect. A total of 13 binomial tests were performed to test whether the percentages of $G p-9^{B B}$ and $G p-9^{B b}$ individuals differed significantly from $50 \%$ for (i) all reared worker pupae, (ii) worker pupae reared by queenless monogyne workers, (iii) worker pupae reared by queenright monogyne workers, (iv) worker pupae reared by queenless polygyne workers, (v) worker pupae reared by queenright polygyne workers, (vi) all reared queen pupae, (vii) queen pupae reared by queenless polygyne workers, (viii) queen pupae reared by queenright polygyne workers (ix) queen pupae reared by queenless monogyne workers (none were reared by queenright monogyne workers), ( $\mathrm{x}$ ) all sampled adult workers, (xi) small adult workers, (xii) medium adult workers and (xiii) large adult workers.
Binomial tests were performed in $\mathrm{R}$ version 2.5.2 ( $\mathrm{R}$ Development Core Team 2012), and linear models were run in IBM SPSS for Mac OSX version 19. The linearity assumption is met for all three models, and overdispersion was accounted for by including an additional random factor at the level of individual observation (Bolker et al. 2009). All errors given represent one standard error.

\section{Results}

$G p-9^{B B}$ queen pupae but not $G p-9^{B B}$ worker pupae are underrepresented

Under Mendelian segregation, a $G p-9^{B b}$ queen mated to a $G p-9^{B}$ male should produce $50 \% \quad G p-9^{B B}$ and $50 \%$ $G p-9^{B b}$ offspring. There was a significant deviation from these expected values for queen pupae with only $23.0 \pm 11.0 \%$ having the $G p-9^{B B}$ genotype (binomial test: $n=336, \mathrm{Gp}-9 \mathrm{Bв}=81, P<0.001)$. The deviation from $50 \%$ was significant in each of the three treatments (queenless polygyne workers, queenless monogyne workers and queenright polygyne workers; binominal tests: $P \leq 0.001$ for each test, see Table 1, Fig. 1).

By contrast, there was no significant deviation from the expected distribution of $50 \% \mathrm{Gp}-9^{B B}$ and $50 \% \mathrm{Gp}-9^{B b}$ worker pupae. This was the case for all treatments considered together (binomial test: $n=561, \mathrm{Gp}-9^{\mathrm{BB}}=278$, $P=0.43$ ) and for each of the four individual treatments (queenless polgygyne workers, queenless monogyne workers and queenright monogyne workers and queenright polygyne workers: binomial tests: $P \geq 0.25$ for all tests, see Table 1, Fig. 2). Furthermore, the proportion of $G p-9^{B B}$ among worker pupae was affected neither by social form of workers in the recipient colonies (GLMMs: $F=1.086$, d.f. $=1,25, P=0.30$, Fig. 2 ) nor by queen presence/absence (GLMMs: $F=0.531$, d.f. $=1,25$, $P=0.47$, Fig. 2).

Table 1 Binomial tests on the frequencies of $G p-9 B B$ female pupae tested against the expected frequency of 0.5 under Mendelian segregation. Shown are the proportions of $G p-9 B B$ pupae reared by workers of the monogyne and polygyne social form under queenright and queenless conditions and the total proportions of $G p-9 B B$ pupae genotyped in this study

\begin{tabular}{|c|c|c|c|c|c|c|c|c|}
\hline Caste & $\begin{array}{l}\text { Social form of } \\
\text { rearing workers }\end{array}$ & $\begin{array}{l}\text { Queen } \\
\text { presence }\end{array}$ & Colonies & $\begin{array}{l}\text { Total } \\
\text { genotyped }\end{array}$ & $\begin{array}{l}\text { Total } \\
\text { Gp-9BB }\end{array}$ & $\begin{array}{l}\text { Expected frequency } \\
\text { of } G p-9 B B\end{array}$ & $\begin{array}{l}\text { Observed frequency } \\
\text { of } G p-9 B B\end{array}$ & $P$ \\
\hline Worker & Monogyne & Present & 4 & 41 & 19 & 0.5 & 0.46 & 0.38 \\
\hline Worker & Polygyne & Present & 4 & 65 & 30 & 0.5 & 0.46 & 0.31 \\
\hline Worker & Monogyne & Absent & 10 & 227 & 108 & 0.5 & 0.47 & 0.25 \\
\hline Worker & Polygyne & Absent & 10 & 228 & 121 & 0.5 & 0.53 & 0.84 \\
\hline Queen & Polygyne & Present & 5 & 211 & 54 & 0.5 & 0.26 & $<0.001$ \\
\hline Queen & Monogyne & Absent & 3 & 53 & 15 & 0.5 & 0.28 & 0.001 \\
\hline Queen & Polygyne & Absent & 3 & 72 & 12 & 0.5 & 0.17 & $<0.001$ \\
\hline Total workers & & & & 561 & 278 & 0.5 & 0.5 & 0.43 \\
\hline Total queens & & & & 336 & 81 & 0.5 & 0.24 & $<0.001$ \\
\hline
\end{tabular}




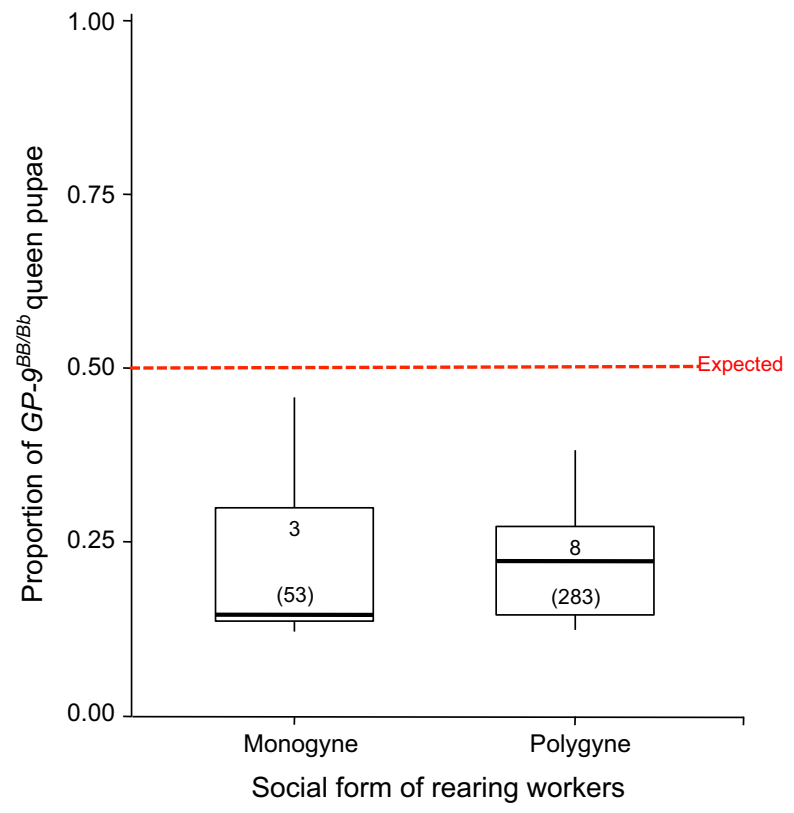

Fig. 1 The proportion of $G p-9^{B B / B b}$ queen pupae reared by unrelated workers of the polygyne social form and monogyne social form under queenless and queenright conditions. Box plot showing median (line), 25-75\% quartiles (boxes) and $1.5 \times$ interquartile ranges (whiskers). The number in each box represents the number of tested colonies with numbers of genotyped queen pupae given in parentheses. The dashed line indicates the $50 \% \mathrm{G} p-9^{B B}$ queen pupae expected under Mendelian segregation.

$G p-9^{B B}$ workers are underrepresented among medium and large workers

Because there were equal proportions of $G p-9^{B B}$ and $G p-9^{B b}$ worker pupae, we expected to also find an equal proportion of both genotypes among adult workers. However, this was not the case. There was a significant underrepresentation of $G p-9^{B B}$ workers in comparison to $G p-9^{B b}$ workers $(43.8 \pm 2.5 \% \quad B B$ overall; binomial test: $\left.n=744, \mathrm{Gp}-9^{\mathrm{BB}}=326 ; P<0.001\right)$. Moreover, the proportion of $G p-9^{B B}$ workers decreased as worker size increased (GLMMs: $F=4.279$, d.f. $=2,31, \quad P=0.02$, Fig. 3). We found $50 \% \mathrm{Gp}-9^{B B}$ individuals among small workers (binomial test: $n=134, G p-9^{B B}=68, P=0.60$ ), $46 \%$ among medium workers (binomial test: $n=365$, $\left.G p-9^{B B}=168, \quad P=0.07\right)$ and only $36 \%$ among large workers (binomial test: $n=245, G p-9^{B B}=90, P<0.001$ ). As there was a higher probability of successfully genotyping larger workers than smaller workers (see Methods) and because there was a lower proportion of $G p-9^{B B}$ individuals among large workers, this could potentially bias our estimate of the overall worker genotype frequencies. We therefore conducted resampling analyses of our data by controlling for the sampling proportions of small (0.20), medium (0.53) and large

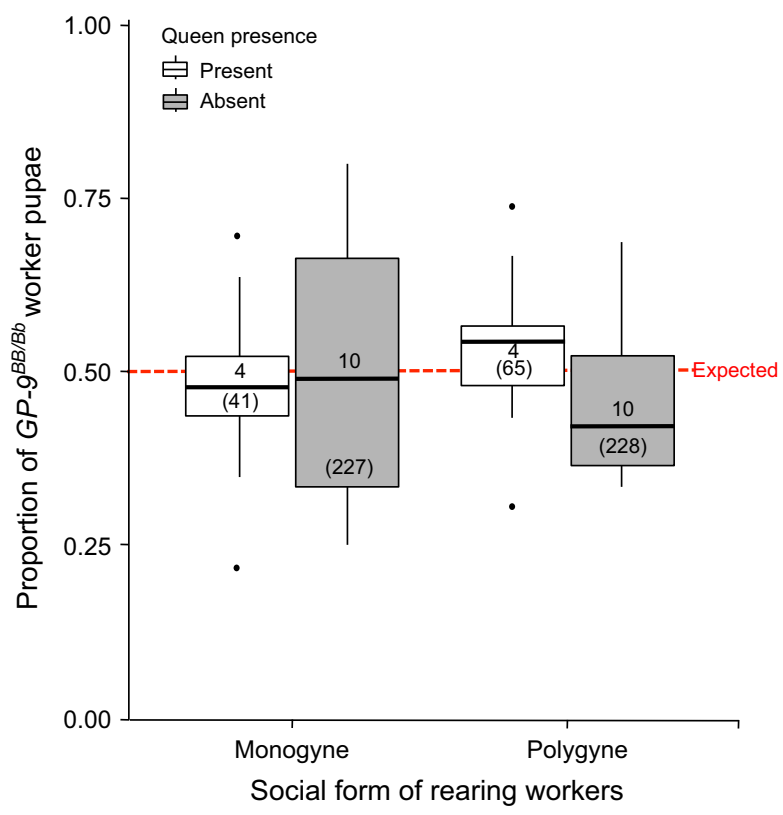

Fig. 2 The proportion of $G p-9^{B B / B b}$ worker pupae reared by unrelated workers of the polygyne social form and monogyne social form, under queenless (white boxes) and queenright conditions (grey boxes). Box plot showing median (line), 25-75\% quartiles (boxes) and $1.5 \times$ interquartile ranges (whiskers). The number in each box represents the number of tested colonies with numbers of genotyped worker pupae given in parentheses. The dashed line indicates the $50 \% \quad G p-9^{B B}$ worker pupae expected under Mendelian segregation.

(0.27) workers (Fig. 4). In each of ten such re-analyses, we found a significant underrepresentation of $G p-9^{B B}$ workers in comparison to $G p-9^{B b}$ workers $(44.4 \pm 0.3 \%$, all $P<0.004)$ Thus, the overall underrepresentation of the $G p-9^{B B}$ genotype among adult workers was not due to a sample bias in the process of genotyping.

More workers were found inside the nest than outside the nest $(60 \%$ of adult workers were collected inside the nest and $40 \%$ outside the nest, GLMMs: $F=12.784$ d.f. $=1,30, P=0.001)$, but there was no significant difference in worker size distribution between the two sampling locations (GLMMs: size-location interaction effect $F=1.897$ d.f. $=2,30, P=0.17$ ). Similarly, the proportion of $G p-9^{B B}$ workers did not vary significantly according to the location (inside vs. outside the nest; GLMMs: $F=1.585$, d.f. $=1,31, P=0.22$ ).

\section{Discussion}

We found that $G p-9^{B b}$ queens mated to single $G p-9^{B}$ males rear equal proportions of $G p-9^{B B}$ and $G p-9^{B b}$ worker pupae. This suggests that $G p-9^{B}$ and $G p-9^{b}$ segregate in a Mendelian manner and that there is no intrinsic difference in viability between $G p-9^{B B}$ and 


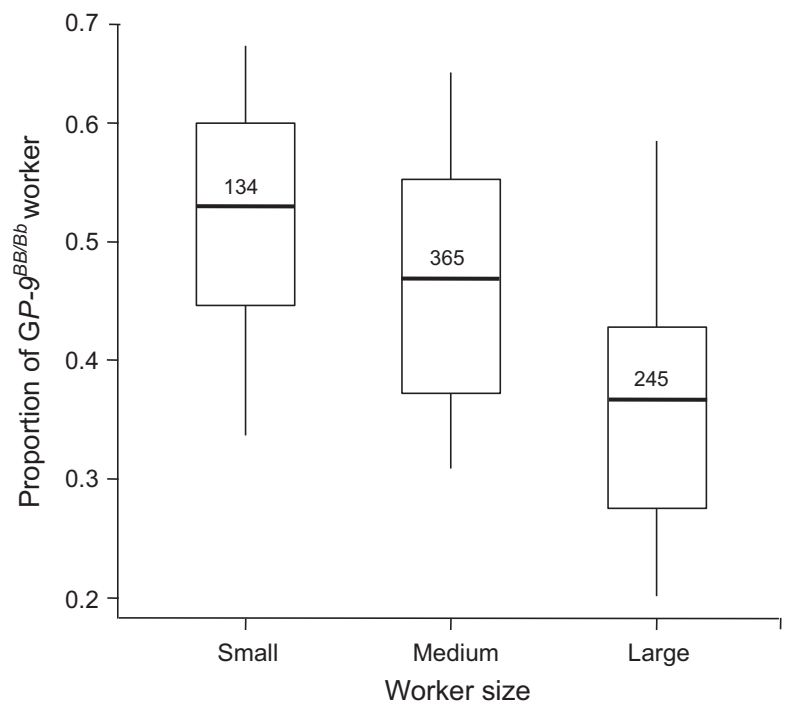

Fig. 3 The proportion of $G p-9^{B B / B b}$ for small, medium and large workers, sampled over six polygyne colonies reared under laboratory conditions. Box plot showing median, (line) 25-75\% quartiles (boxes) and $1.5 \times$ interquartile ranges (whiskers). The number in each box represents the number of workers that were genotyped.

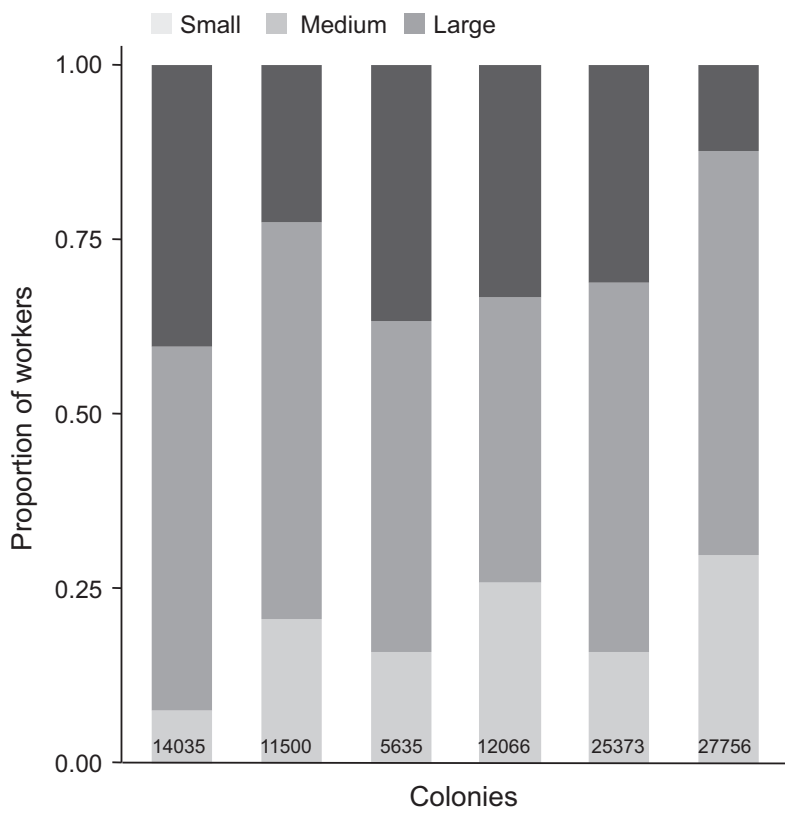

Fig. 4 The proportion of small, medium and large workers in six polygyne colonies reared under laboratory conditions. Shades of grey represent worker size; light grey: small workers, medium grey: medium workers and dark grey: large workers. The combined weight of all ants in each category was used to estimate the respective total numbers of ants per colony (small numbers) and their overall proportions.

$G p-9^{B b}$ workers until the pupal stage. However, for queen pupae, there was a dramatic underrepresentation of $G p-9^{B B}$ individuals in comparison with $G p-9^{B b}$ individuals. The design of this study allows one to rule out the hypothesis that $G p-9^{B B}$ queens develop more slowly than queens of the alternate genotype and our result being an artefact of sampling (i.e. if only the first pupae to develop were sampled while most of the Gp$9^{B B}$ pupae were still in the larval stage). The collection in the queenless colonies was performed on day 28, at the peak of production of queen pupae and we therefore did not collect the first pupae produced. Moreover, in the queenright colonies, sampling occurred over 3 weeks and thus over several generations of pupae (the pupal stage is about 8-9 days under our laboratory conditions).

There are three possible explanations for the dramatic underrepresentation of the $G p-9^{B B}$ genotype among queen pupae. The first is that workers actively eliminate $G p-9^{B B}$ females when these develop into queens. This process would mirror the ability of $G p-9^{B b}$ workers to detect and selectively execute $G p-9^{B B}$ queens when they reach sexual maturity (Keller \& Ross 1998). However, the finding of similar underrepresentation of $G p-9^{B B}$ queen pupae in colonies that contained only $G p-9^{B B}$ monogyne workers indicates that underrepresentation of $G p-9^{B B}$ queen pupae cannot be due to their selective killing by $G p-9^{B b}$ workers. Hence, a selective elimination of $G p-9^{B B}$ queen pupae could only occur if $G p-9^{B B}$ workers would also discriminate against queens of their own genotype. It is not easy to see what selective force may have led to such a scenario.

The second possible explanation is higher intrinsic mortality of queen-destined $G p-9^{B B}$ females compared to queen-destined $G p-9^{B b}$ females. This scenario would be surprising given the evolutionary history of the genomic region containing $G p-9$. $G p-9^{B B}$ females have two $G p-9^{B}$ social chromosomes (SB), while $G p-9^{B b}$ females have one $\mathrm{SB}$ and one $\mathrm{G} p-9^{b}$ social chromosome (Sb). While the SB chromosomes can recombine in SB/SB females, a large part (more than $60 \%$ ) of the $\mathrm{Sb}$ chromosome is thought to never recombine because it cannot recombine with the SB chromosome (Wang et al. 2013) and because $\mathrm{Sb} / \mathrm{Sb}$ individuals are not viable (Hallar et al. 2007). The large nonrecombining part of $\mathrm{Sb}$ is expected to degenerate similarly to a Y chromosome (Wang et al. 2013). Consistent with this view, $\mathrm{Sb}$ contains many more transposable elements and other repetitive elements compared to SB (Wang et al. 2013). Moreover, the nonviability of $\mathrm{Sb} / \mathrm{Sb}$ females is consistent with the presence of one or several deleterious recessive elements in the nonrecombining region of $\mathrm{Sb}$ (Hallar et al. 2007). Thus, if anything, one would predict lower viability of $G p-9^{B b}$ queen pupae rather than lower viability of $G p-9^{B B}$ queen pupae. Moreover, the idea of differential viability of queen pupae would be somewhat surprising given that no such effect was found for worker pupae. 
Finally, the third and most plausible explanation is that one or several genes in linkage disequilibrium with the $G p-9^{b}$ allele increase the likelihood of female brood to develop into queens. Genetic effects on caste determination have been recently reported in various ant species (Schwander et al. 2010). The presence of allelic variants affecting caste determination in the Sb chromosome would be consistent with this haplotype being a selfish genomic region acting in a manner analogous to segregation distortion alleles which have a higher than $50 \%$ chance to be in the gametes (Sandler \& Novitski 1957). This should lead to a corresponding drop in the portion of $G p-9^{B b}$ individuals among worker pupae. However, because most females develop into workers rather than queens, a threefold to fourfold greater likelihood of $G p-9^{B b}$ females to develop into queens compared to $G p-9^{B B}$ females (a value consistent with the queen pupae genotype data) would only lead to relatively small and thus undetectable genotype bias in workers. In our experiments, only 211 females developed into queens while there were many thousands workers produced during the 3 weeks of sampling.

Ultimately and from the point of view of a selfish genomic element spreading through the population, the social chromosome variant $\mathrm{SB} / \mathrm{Sb}$ promotes queen development rather than worker development and thereby helps the spread of $G p-9^{b}$ in the next generations. This is because some queens with $G p-9^{B B}$ genotype evade the aggression of $G p-9^{B b}$ workers and disperse to found monogyne colonies (DeHeer et al. 1999). This bias in caste determination, hence, has significant implications for the population dynamics of the two social chromosome variants in fire ants populations containing both monogyne and polygyne nests.

Our experiments also revealed that $G p-9^{B B}$ individuals were underrepresented among adult workers. While there was an equal representation of $G p-9^{B B}$ and $G p-9^{B b}$ individuals among small workers, $G p-9^{B B}$ individuals were underrepresented among medium-sized and large workers, confirming field-based data showing lower frequencies of $G p-9^{B B}$ workers than expected from the genotypes of their parents (Ross 1997; Goodisman et al. 2000b; Fritz 2006). The finding of a greater proportion of $G p-9^{B b}$ individuals among larger workers than smaller workers could be explained if $G p-9^{B b}$ females were more likely to develop into larger workers than $G p-9^{B B}$ individuals. This would mirror the finding of $G p-9^{B b}$ females having a higher probability than $G p-9^{B B}$ females to develop into queens possibly because female $G p-9^{B b}$ larvae can secure more food than $G p-9^{B B}$ larvae. In most ants, the larval development pathway is influenced by the type and amount of food ingested. For example, larvae that develop into larger castes were shown to feed on resources with higher nitrogen content, most likely insect prey, compared to larvae that develop into smaller castes (Smith et al. 2008). But while altered larval nutrition could impact the size and caste determination of $G p-9^{B b}$ larvae, the exact mechanism underlying our findings remains to be identified.

If workers with a $G p-9^{B b}$ genotype were more likely to develop into large workers, this should lead to this genotype being underrepresented among small workers, which was not the case. Given that large workers represented only $27 \%$ of the workers and that they have about twice the lifespan of small workers (Calabi \& Porter 1989), it is possible that the developmental bias is relatively weak and not sufficient for the percentage of $G p-9^{B b}$ individuals among small workers to deviate significantly from 50\%. Alternatively, and most likely, the overrepresentation of the $G p-9^{B b}$ genotype among workers, and large workers in particular, may reflect increased mortality of large workers with the $G p-9^{B B}$ genotype. This may occur if large workers would have an odour profile similar to that of queens, inducing $G p-9^{B b}$ workers to eliminate large $G p-9^{B B}$ workers in the same way as they selectively eliminate $G p-9^{B B}$ queens (Keller \& Ross 1998). However, the lack of supporting data renders this statement currently speculative. Finally, it may also be possible that the observed genotypic frequencies could be explained if $G p-9^{B B}$ workers have lower longevity than $G p-9^{B b}$ workers and if there also is a bias for $G p-9^{B b}$ workers to preferentially develop into larger workers. In this scenario, the lack of deviation from $50 \%$ frequency for small workers would be a product of both effects.

The finding of an underrepresentation of mediumsized and large $G p-9^{B B}$ workers is interesting with regards to the observation that workers with this genotype are underrepresented in the field compared to the values expected on the basis of the genotypes of the reproductive individuals. The observed underrepresentation of such workers in the field varied among field studies (6\% Ross 1997; 15-25\% Goodisman et al. 2000b; 5-18\% Fritz 2006); such underrepresenation was lower in our laboratory study $(0-11 \%$, see also Table S1, Supporting information). Three lines of reasoning may explain the differences between studies. The first is that stress leading to extrinsic life expectancy differences between workers with different $G p-9$ genotypes may be greater in the field than in the laboratory. Consistent with this view, Goodisman et al. (2007) found an effect of sampling date on the frequency of $G p-9^{B B}$ and $G p-9^{B b}$ individuals in the field, hence suggesting the rate of mortality among alternate genotypes may vary according to temperature or other extrinsic factors. Alternatively, there could be sampling biases in the field with larger workers being more likely to be sampled than smaller workers (see Goodisman et al. 2007 for a similar 
argumentation). Such a bias would lead to the proportion of $G p-9^{B b}$ workers being overestimated in previous field studies. Finally, there could also be variation in the spatial distribution of $G p-9^{B B}$ and $G p-9^{B b}$ workers. A recent study indeed found a greater proportion of $G p-9^{B B}$ than $G p-9^{B b}$ workers in the foraging arena compared to the nest (C. Lucas, M. Nicolas \& L. Keller, unpublished). In the present study, there was no significant difference between genotypic distributions inside and outside the nest, possibly because the nest was not clearly separated from the foraging arena. In the Lucas et al. study, which was specifically designed to test for an effect of location on genotypic frequencies, the foraging arena was separated from the nest by a 1-metrelong tube. Thus, spatial segregation may also contribute to the greater discrepancy between expected and observed proportion of $G p-9^{B b}$ workers in field studies (where worker location was not controlled for) compared to our study where a possible effect of worker location was controlled for while sampling.

In conclusion, our study revealed that the nonrecombining region of the two fire ant social chromosome variants affects development of female larvae, leading to a strong bias towards $\mathrm{SB} / \mathrm{Sb}$ queen pupae in comparison with $\mathrm{SB} / \mathrm{SB}$ queen pupae, while here was no genotypic bias among worker pupae suggesting that the presence of the $\mathrm{Sb}$ chromosome may bias female development towards queen differentiation. This has significant fitness implications for the two variants of social chromosomes. A previous study (DeHeer et al. 1999) showed that only relatively few SB/SB queens depart on mating flights from polygyne colonies. This together with the selective elimination by $\mathrm{SB} / \mathrm{Sb}$ workers of $\mathrm{SB} / \mathrm{SB}$ queens when they initiate reproduction acts as potent mechanism to favour the $\mathrm{Sb}$ variant at the population level. Our study also revealed an underrepresentation of SB/SB workers among medium-sized and large workers, which together with sources of sampling bias may account for similar findings reported from the field. Overall, our study thus shows that the alternate social chromosomes have widespread phenotypic effects not only in adults but also in immature S. invicta females.

These findings are interesting in relation to the increasing number of discoveries of supergenes which affects traits as diverse as ecotypes in monkeyflowers (Lowry \& Willis 2010), self-incompatibility in angiosperms (Takayama \& Isogai 2005), female morphs in cichlid fish (Roberts et al. 2009), wing pattern mimicry morphs in butterflies (Joron et al. 2011), plumage polymorphism in birds (Huynh et al. 2010) and shell colour polymorphism in snails (Cook 1998). The evolution of such supergenes likely involves selection for reduced recombination between loci that epistatically lead to higher overall fitness (Turner 1967). When this occurs, mildly deleterious mutations and neighbouring genes with unrelated effects can become part of a supergene as a result of hitchhiking (Smith \& Haigh 1974) or supergene expansion (Charlesworth \& Charlesworth 1975). Furthermore, mildly deleterious mutations can accumulate within established supergenes due to Mueller's ratchet (Mueller 1964). Because each gene within the supergene region can pleotropically affect multiple phenotypic traits (Stearns 2010), supergenes can be expected to have overall complex phenotypic effects including some that are deleterious. At the most extreme level, homozygosity of some supergene variants can be lethal [e.g. many Y chromosomes (Bachtrog 2013), t-haplotypes in mice (Lyon 2003), Sb in fire ants (Ross 1997; Keller \& Ross 1999; Hallar et al. 2007; Wang et al. 2013)]. However, only few studies have empirically demonstrated that supergenes have complex effects on phenotypes at various stages of development. Our study is thus valuable because it demonstrates that the social chromosomes in S. invicta do not only influence the phenotypes of adult queens and males (Goodisman et al. 1999; Lawson et al. 2012) and the behaviours of queens and workers (Keller \& Ross 1998; DeHeer et al. 1999; DeHeer 2002; Krieger 2005; Goodisman et al. 2007) but also the processes of caste determination and the survival of SB/SB and SB/Sb workers. A challenge will be to determine which of the $>600$ genes present in the social chromosomes are responsible for this large suite of phenotypic effects and determine how these phenotypic differences have accumulated during the course of evolution.

\section{Acknowledgements}

We thank M. Nipitwattanaphon, M. Dijkstra and K.G. Ross for collecting ants, C. Lucas and M. Nicolas, J. Wang and D.D. Shoemaker for advice on Gp-9 genotyping, C. LaMendola and C. Stoeffel for genotyping adults and sexuals ants, M. Nipitwattanaphon and D. Gotzek for helpful advice on Solenopsis rearing and handling, J. Notari, J. Rufener and L. Maumaury for animal care taking, P. Bize, M. Dijkstra and S. Barribeau for statistical advice and A. Kotrschal and the three reviewers for very useful comments and suggestions on the manuscript. This project was funded by grants from the Swiss NSF and an Advanced ERC Grant.

\section{References}

Aron S, Vargo EL, Passera L (1995) Primary and secondary sex ratios in monogyne colonies of the fire ant. Animal Behaviour, 49, 749-757.

Ascunce MS, Bouwma AM, Shoemaker DD (2009) Characterization of 24 microsatellite markers in 11 species of fire ants in the genus Solenopsis (Hymenoptera: Formicidae). Molecular Ecology Resources, 9, 1475-1479. 
Bachtrog D (2013) Y-chromosome evolution: emerging insights into processes of Y-chromosome degeneration. Nature Reviews Genetics, 14, 113-124.

Bolker BM, Brooks ME, Clark CJ et al. (2009) Generalized linear mixed models: a practical guide for ecology and evolution. Trends in Ecology \& Evolution, 24, 127-135.

Bradshaw JWS, Howse PE (1984) Sociochemicals of ants. In: Chemical Ecology of Insects (eds Bell WJ, Carde RT), pp. 429473. Springer US, Boston, Massachusetts.

Calabi P, Porter SD (1989) Worker longevity in the fire ant Solenopsis invicta: ergonomic considerations of correlations between temperature, size and metabolic rates. Journal of Insect Physiology, 35, 643-649.

Charlesworth D, Charlesworth B (1975) Theoretical genetics of Batesian mimicry II. Evolution of supergenes. Journal of Theoretical Biology, 55, 305-324.

Cook L (1998) A two-stage model for Cepaea polymorphism. Philosophical Transactions of the Royal Society of London Series B: Biological Sciences, 353, 1577-1593.

Crawley M (1993) GLIM for Ecologists. Wiley-Blackwell, Cambridge, UK.

DeHeer CJ (2002) A comparison of the colony-founding potential of queens from single- and multiple-queen colonies of the fire ant Solenopsis invicta. Journal of Theoretical Biology, 64, 655-661.

DeHeer CJ, Goodisman MAD, Ross KG (1999) Queen dispersal strategies in the multiple-queen form of the fire ant Solenopsis invicta. The American Naturalist, 153, 660-675.

Dillon N, Austin AD, Bartowsky E (1996) Comparison of preservation techniques for DNA extraction from hymenopterous insects. Insect Molecular Biology, 5, 21-24.

Fritz GN (2006) Selective male mortality in the red imported fire ant, Solenopsis invicta. Genetics, 173, 207-213.

Goodisman MAD, Mack PD, Pearse DE et al. (1999) Effects of a single gene on worker and male body mass in the fire ant Solenopsis invicta (Hymenoptera: Formicidae). Annals of the Entomological Society of America, 92, 563-570.

Goodisman MAD, DeHeer CJ, Ross KG (2000a) Unusual behavior of polygyne fire ant queens on nuptial flights. Journal of Insect Behavior, 13, 455-468.

Goodisman MAD, Ross KG, Asmussen MA (2000b) A formal assessment of gene flow and selection in the fire ant Solenopsis invicta. Evolution, 54, 606-616.

Goodisman MAD, Sankovich KA, Kovacs JL (2007) Genetic and morphological variation over space and time in the invasive fire ant Solenopsis invicta. Biological Invasions, 9, 571584.

Gotzek D, Ross KG (2008) Experimental conversion of colony social organization in fire ants (Solenopsis invicta): worker genotype manipulation in the absence of queen effects. Journal of Insect Behavior, 21, 337-350.

Hallar BL, Krieger MJB, Ross KG (2007) Potential cause of lethality of an allele implicated in social evolution in fire ants. Genetica, 131, 69-79.

Huynh LY, Maney DL, Thomas JW (2010) Chromosome-wide linkage disequilibrium caused by an inversion polymorphism in the white-throated sparrow (Zonotrichia albicollis). Heredity, 106, 537-546.

Joron M, Frezal L, Jones RT et al. (2011) Chromosomal rearrangements maintain a polymorphic supergene controlling butterfly mimicry. Nature, 477, 203-206.
Jouvenaz DP, Allen GE, Banks WA et al. (1977) A survey for pathogens of fire ants, Solenopsis spp., in the Southeastern United States. The Florida Entomologist, 60, 275-279.

Keller L (1995) Social life: the paradox of multiple-queen colonies. Trends in Ecology \& Evolution, 10, 355-360.

Keller L, Ross KG (1993a) Phenotypic plasticity and "cultural transmission" of alternative social organizations in the fire ant Solenopsis invicta. Behavioral Ecology and Sociobiology, 33, 121-129.

Keller L, Ross KG (1993b) Phenotypic basis of reproductive success in a social insect: genetic and social determinants. Science, 260, 1107-1110.

Keller L, Ross KG (1998) Selfish genes: a green beard in the red fire ant. Nature, 394, 573-575.

Keller L, Ross KG (1999) Major gene effects on phenotype and fitness: the relative roles of $P g m-3$ and Gp-9 in introduced populations of the fire ant Solenopsis invicta. Evolution, 12, 672-680.

Krieger MJB (2005) To b or not to b: a pheromone-binding protein regulates colony social organization in fire ants. BioEssays, 27, 91-99.

Krieger MJB, Keller L (1997) Polymorphism at dinucleotide microsatellite loci in fire ant Solenopsis invicta populations. Molecular Ecology, 6, 997-999.

Lawson LP, Vander Meer RK, Shoemaker DD (2012) Male reproductive fitness and queen polyandry are linked to variation in the supergene Gp-9 in the fire ant Solenopsis invicta. Proceedings of the Royal Society. Biological sciences, 320, 1213-1216.

Lowry DB, Willis JH (2010) A widespread chromosomal inversion polymorphism contributes to a major life-history transition, local adaptation, and reproductive isolation. PLoS Biology, 8, e1000500.

Lyon MF (2003) Transmission ratio distortion in mice. Annual Review of Genetics, 37, 393-408.

Mueller HJ (1964) The relation of recombination to mutational advance. Mutation Research, 106, 2-9.

Nipitwattanaphon M, Wang J, Dijkstra MB et al. (2013) A simple genetic basis for complex social behaviour mediates widespread gene expression differences. Molecular Ecology, 22, 3797-3813.

Passera A, Vargo EL, Keller L (2001) Queen control of sex ratio in fire ants. Science, 293, 1308-1310.

Porter SD (1988) Impact of temperature on colony growth and developmental rates of the ant, Solenopsis invicta. Journal of Insect Physiology, 34, 1127-1133.

R Development Core Team (2012) R: A Language and Environment for Statistical Computing. R Foundation for Statistical Computing, Vienna, Austria.

Roberts RB, Ser JR, Kocher TD (2009) Sexual conflict resolved by invasion of a novel sex determiner in Lake Malawi cichlid fishes. Science, 326, 998-1001.

Ross KG (1997) Multilocus evolution in fire ants: effects of selection, gene flow and recombination. Genetics, 145, 961-974.

Ross KG, Keller L (1995) Ecology and evolution of social organization: insights from fire ants and other highly eusocial insects. Annual Review of Ecology and Systematics, 26, 631-656.

Ross KG, Keller L (1998) Genetic control of social organization in an ant. Proceedings of the National Academy of Sciences of the United States of America, 95, 14232-14237.

Ross KG, Keller L (2002) Experimental conversion of colony social organization by manipulation of worker genotype 
composition in fire ants (Solenopsis invicta). Behavioral Ecology and Sociobiology, 51, 287-295.

Sandler L, Novitski E (1957) Meiotic drive as an evolutionary force. The American Naturalist, 91, 105-110.

Schwander T, Lo N, Beekman M, Oldroyd BP, Keller L (2010) Nature versus nurture in social insect caste differentiation. Trends in Ecology \& Evolution, 25, 275-282.

Schwander T, Libbrecht R, Keller L (2014) Supergenes and complex phenotypes. Current Biology, 24, R288-R294.

Shoemaker DD, Ascunce MS (2010) A new method for distinguishing colony social forms of the fire ant, Solenopsis invicta. Journal of Insect Science, 10, 1-11.

Shoemaker DD, DeHeer CJ, Krieger MJB, Ross KG (2006) Population genetics of the invasive fire ant Solenopsis invicta (Hymenoptera: Formicidae) in the United States. Annals of the Entomological Society of America, 99, 1213-1233.

Smith JM, Haigh J (1974) The hitch-hiking effect of a favourable gene. Genetics Research, 23, 23-35.

Smith CR, Anderson KE, Tillberg CV, Gadau J, Suarez AV (2008) Caste determination in a polymorphic social insect: nutritional, social, and genetic factors. The American Naturalist, 172, 497-507.

Stearns FW (2010) One hundred years of pleiotropy: a retrospective. Genetics, 186, 767-773.

Takayama S, Isogai A (2005) Self-incompatibility in plants. Annual Review of Plant Biology, 56, 467-489.

Thomas JW, Caceres M, Lowman JJ et al. (2008) The chromosomal polymorphism linked to variation in social behavior in the white-throated sparrow (Zonotrichia albicollis) is a complex rearrangement and suppressor of recombination. Genetics, 179, 1455-1468.

Tschinkel WR (2006) The Fire Ants. Belknap Press, Cambridge, Massachusetts and London, UK.

Turner JRG (1967) On supergenes. I. The evolution of supergenes. The American Naturalist, 101, 195-221.
Vargo EL (1996) Sex investment ratios in monogyne and polygyne of the fire ant Solenopsis invicta populations. Journal of Evolutionary Biology, 9, 783-802.

Wang J, Ross KG, Keller L (2008) Genome-wide expression patterns and the genetic architecture of a fundamental social trait. PLoS Genetics, 4, e1000127.

Wang J, Wurm Y, Nipitwattanaphon M et al. (2013) A Y-like social chromosome causes alternative colony organization in fire ants. Nature, 493, 664-668.

S.D.B., Y.W. and L.K. planned the experiments, S.D.B. collected the data, performed the analyses and wrote a first draft, S.D.B., Y.W. and L.K. wrote the manuscript.

\section{Data accessibility}

Gp-9 genotypes of female pupae and adult workers, and data on worker size and worker distribution are deposited in Dryad: doi: 10.5061/dryad.vb073.

\section{Supporting information}

Additional supporting information may be found in the online version of this article.

Table S1 Expected and observed frequencies of the Gp- $9^{B B}$ genotypes of adult workers, queen pupae and worker pupae from two populations of Solenopsis invicta. 